\title{
Correction to: Staging, recurrence and follow-up of uterine cervical cancer using MRI: Updated Guidelines of the European Society of Urogenital Radiology after revised FIGO staging 2018
}

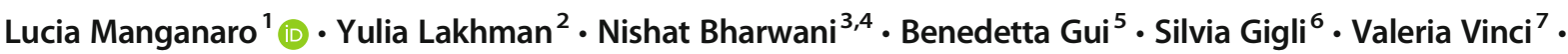 \\ Stefania Rizzo ${ }^{8} \cdot$ Aki Kido $^{9}$ - Teresa Margarida Cunha ${ }^{10}$. Evis Sala ${ }^{11}$. Andrea Rockall ${ }^{12,13}$. Rosemarie Forstner $^{14}$. \\ Stephanie Nougaret ${ }^{15,16}$ \\ Published online: 17 June 2021 \\ (C) European Society of Radiology 2021
}

\section{Correction to: European Radiology}

https://doi.org/10.1007/s00330-020-07632-9

The original version of this article, published on 14 April 2021, unfortunately contained a mistake. The following correction has therefore been made in the original: Affiliation 1

The online version of the original article can be found at https://doi.org/ 10.1007/s00330-020-07632-9

Lucia Manganaro

lucia.manganaro@uniroma1.it

1 Department of Radiological, Oncological and Pathological Sciences, Sapienza University of Rome, Viale Regina Elena, 32400161 Rome, Italy

2 Department of Radiology, Memorial Sloan Kettering Cancer Center, 1275 York Avenue, New York, NY 10065, USA

3 Department of Surgery and Cancer, Imperial College, London, UK

4 Department of Radiology, St Mary's Hospital, Imperial College Healthcare NHS Trust, Department of Surgery and Cancer, Imperial College, 3rd Floor, Queen Elizabeth the Queen Mother Building, Praed St W21NY, London, UK

5 Fondazione Policlinico Universitario A. Gemelli IRCCS, UOC Radiologia Generale ed Interventistica Generale, Area Diagnostica per Immagini, Dipartimento Diagnostica per Immagini, Radioterapia Oncologica ed Ematologia, Rome, Italy

6 Department of Diagnostic Imaging, Sandro Pertini Hospital, Via dei Monti Tiburtini, 385, 00157 Rome, Italy

7 Department of Radiology, Aerospace Medical Institute, Viale Piero Gobetti, Rome, Italy was incorrect. The corrected affiliation is given below. The original article has been corrected.

Publisher's note Springer Nature remains neutral with regard to jurisdictional claims in published maps and institutional affiliations.
8 Department of Radiology, Università della Svizzera Italiana (USI), EOC via Tesserete 46, 6900 Lugano, Switzerland

9 Department of Diagnostic Radiology and Nuclear Medicine, Kyoto University Hospital, 54 Shogoin-Kawaharacho, Sakyo-ku, Kyoto 606-8507, Japan

10 Department of Radiology, Instituto Português de Oncologia de Lisboa Francisco Gentil, R. Prof. Lima Basto, 1099-023 Lisbon, Codex, Portugal

11 Department of Radiology, Cambridge Biomedical Campus Cambridge, Box 218, Cambridge CB2 0QQ, UK

12 Department of Surgery and Cancer, Faculty of Medicine, Imperial College, London, UK

13 Department of Radiology, Imperial College Healthcare NHS Trust, London, UK

14 Department of Radiology, PMU, Müllner Hauptstr, 48 Universitätsklinikum, 5020 Salzburg, Austria

15 Department of Radiology, University of Montpellier, Montpellier, France

16 INSERM, Montpellier Cancer Research Institute, U1194, University of Montpellier, 208 Avenue des Apothicaires, 34295 Montpellier, France 